\title{
The Key Techniques of Constructing the Database of Treatment Measures for Hidden Troubles in Electric Power System
}

\author{
Shuchun Wang, Xiaoguang Sun, Jianyu Geng, Yuan Han, Chunyong Zhang and Weihua Zhang* \\ Changchun Power Supply Company, State Grid Jilin Electric Power Co., Ltd. Changchun, Jilin 130000, China
}

\begin{abstract}
This article adopts a research method that combined with theoretical analysis and system design, analyzes multiple dimensions of common safety hazard events, uses support vector machine classification algorithms to filter and mine valuable information in massive data, and establishes a common safety hazard event feature set. This kind of technology can realize the automatic classification of safety hazards and extract their characteristic information, introduce the semantic analysis, word segmentation clustering method to determine the typical processing measures of events, and generate knowledge items to add methods and models to the processing measures database, which can solve the grid safety hazard data entry specifications, In-depth analysis and other issues.
\end{abstract}

\section{Introduction}

In recent years, big data concepts and methods have attracted widespread attention in the intelligence community, and scholars generally believe that it has an impact on intelligence research and brings huge opportunities for intelligence studies' development [1]. Under the background of big data, the field of information science needs to be researched; constructed information analysis tools of this discipline; integrated the ideas of information research; applied new information technologies and advanced theoretical and technical methods in other disciplines; also, we should pay attention to embody the intelligence and visualization concepts of big data[2].

In the preliminary exploration, this paper proposes a database of hidden safety measures driven by big data. Over the years, the safety hazard module in the integrated safety and supervision platform system has completed the unified management of safety hazards; standardized the hidden danger information process of reporting, approval, governance, and acceptance [3]. According to the source of hidden dangers, the database of hidden safety measures driven by big data can perform statistical analysis, units of hidden dangers, and classification of hidden hazards.[4].Based on these performances, it is becoming an important platform for the supervision and inspection of various units' works about investing and governing hidden troubles [5]. This platform has accumulated a large amount of data about hidden dangers in power grids, while the following problems exist:
- The data entry of hidden dangers is not standardized, and the semantic expression is inconsistent with each other;

- In-depth analysis methods are in deficiency;

- Data retrieval methods are insufficient, and the method of multi-dimensional retrieval 、 real-time retrieval cannot be achieved together;

- The degree of visual analysis of big data is not enough to directly show the internal laws of the data.

This paper aims at constructing the knowledge bases for processing measures of hidden safety hazards, in which semantic analysis and word segmentation clustering methods are used to determine representative processing measures for events to process large-scale data resources. This is a key technology for constructing intelligence researches and knowledge bases [6]. The following parts will be discussed and implemented two involved technologies by taking the knowledge base of power system safety hazard treatment measures as an example[7].

\section{Domain selection and data acquisition for knowledge base construction}

In order to make key technology research and implementation targeted and feasible, this article will first select the research field and obtain relevant data, and then combine the selected field to demonstrate the research and implementation of key technologies[8]. When choosing a field, this paper mainly considers the

*Corresponding author e-mail: 798291078@qq.com 
emerging or continuity of technology development in it; the degree of attention by scholars, the industry, basic research status and society; the scope of application in this field, and the time span. Based on above considerations and reviewing our country's industrial development policies, it is determined to select the power system as the research field[9].

Safe production is one of the most important foundations of enterprise 、 economic and social development, especially for power grid safety [10]. The safety supervision works and management modes of the power industry are important which links to the entire power development enterprise, agriculture, and agricultural development. The development of the national economy cannot be ignored in the safety supervision and management of the power industry. A wide comprehensive application management system is the main responsible for implementing the development of the power industry and a means to ensure the smooth development of the power industry. The large-scale power outages of power grids caused by power accidents have huge impacts and losses on modern society, and may even bring catastrophic consequences[11]. Therefore, efforts must be made to implement effective precautions, minimize the risk of power grid security, effectively protect the safety of human life, and provide a safe、reliable electrical energy for the rapid development of the social economy[12].

In terms of data acquisition, the data of the integrated safety is used. After removing irrelevant data, 140 hidden danger data and governance fund data of 7 power supply companies are obtained.

\section{Key technologies for knowledge base construction}

\subsection{Establish a common set of hidden safety hazards}

Because the collected data contains a variety of heterogeneous data, data sharing cannot be directly achieved, so ETL (Extract, Transformation, Loading) can be used to complete the conversion between the data. ETL is a key link for data warehouses to obtain high-quality data. It is a process of extracting, converting, cleaning, and loading existing data scattered in various business systems to make these data useful data required by the system. ETL is the first but important step in building a data warehouse[13] . It is roughly divided into: data extraction, data conversion, data cleaning and data loading.

In the ETL process, extraction can be regarded as a data input process, which mainly solves the heterogeneous problem of data sources, that is, extracting data from multiple data sources into a unified data storage, and loading data can be regarded as data. The output process is to load the processed data from the unified data storage into the target data warehouse. The conversion and cleaning between the two mainly solve the problem of data quality. It detects and corrects the redundancy, data errors, and missing data in the massive data through a series of cleaning processes, and uses the default or user-defined Conversion rules to perform operations such as merging and converting certain fields in the data, so that the data can be in good correctness, consistency, integrity, and availability.

The working principle of ETL is to obtain heterogeneous data from multiple systems and extract it to the data interface, and then convert the data to the ideal data format and structure. The conversion process can define some conversion rules, and then call these conversion rules, Finally, the normalized data is loaded into the target system.

\subsection{Propose a classification model of hidden dangers based on big data}

\subsubsection{Classification algorithm based on support} vector machine. The Support Vector Machine (SVM) method's operating principle is to map the sample space to a high-dimensional or even infinite-dimensional feature space (Hilbert space) through a nonlinear mapping $\mathrm{p}$, so that the nonlinearity in the original sample space can be The problem of division is transformed into a linearly separable problem in the feature space[14].

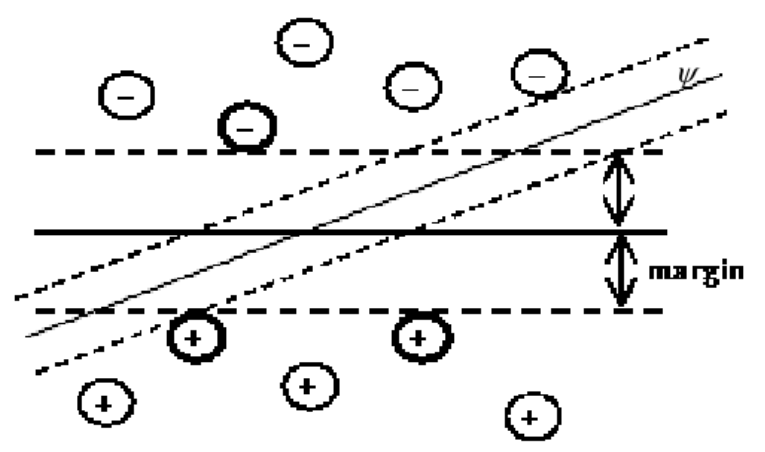

Figure 1. Optimal segmentation plane in feature space

As shown in Figure 1, consider the problem of using a hyperplane in a feature space to perform binary classification on a given training data set. For a given sample point:

$$
\left(x_{1}, y_{1}\right), \ldots,\left(x_{l}, y_{l}\right), x_{i} \in R^{n}, y_{i} \in\{-1,+1\}
$$

The vector ${ }^{x_{i}}$ may be a vector directly constructed by extracting certain features from the object sample set, or it may be a mapping vector in which the original vector is mapped into the kernel space through a kernel function.

Construct the segmentation plane in the feature space:

$$
(w \cdot x)+b=0
$$

So that: 


$$
\left\{\begin{array}{cl}
\left(w \cdot x_{i}\right)+b \geq 1 & y_{i}=1 \\
\left(w \cdot x_{i}\right)+b \leq-1 & y i=-1 \\
& (i=1,2, \ldots, l)
\end{array} \Leftrightarrow y_{i}\left[\left(w \cdot x_{i}\right)+b\right] \geq 1\right.
$$

It can be calculated that the minimum distance from the training data set to a given segmentation plane is:

$$
p(w, b)=\min _{\left\{x_{i} \mid y_{i}=1\right\}} \frac{w \cdot x_{i}+b}{|w|}-\max _{\left\{x_{i} \mid y_{i}=-1\right\}} \frac{w \cdot x_{i}+b}{|w|}=\frac{2}{|w|}
$$

According to the definition of the optimal segmentation plane by SVM, it can be seen that the problem of solving the plane can be simplified as: when the conditional expression (3) is satisfied, the normal vector $w$ and offset $b$ of the segmentation plane that can be maximized $p(w, b)$ are calculated. Vapnik et al. prove:

The normal vector of the hyperplane is a linear combination of all training set vectors. That can be described as:

$$
w_{0}=\sum_{i=1}^{l}\left(a_{i}^{0} y_{i}\right) x_{i} \quad\left(a_{i}^{0} \geq 0\right), i=1, \ldots, l
$$

Define discriminant function

$$
f(x)=w_{0} \cdot x+b_{0}
$$

Then the classification function of the test set can be described as:

$$
\operatorname{label}(x)=\operatorname{sgn}(f(x))=\operatorname{sign}\left(w_{0} \cdot x+b_{0}\right)
$$

It can be seen from (3) that in the case of linear separability, all training samples should satisfy $|f(x)| \geq 1$. In the following, the region satisfying $|f(x)|<1$ is called the boundary region corresponding to the segmented hyperplane.

In most cases (2-18) in the expansion of Equation $w_{0}$, the coefficient $a_{i}^{0}$ has a zero value, and the $a_{i}^{0}$ corresponding to the non-zero value of $x_{i}$ is called the support vector SV. These vectors fully describe the characteristics of the entire training data set, so that the linear division of the $\mathrm{SV}$ set is equivalent to the division of the entire data set.

It can be seen from equation (2-4) that the solution of the optimal segmentation plane is equivalent to maximizing the following equation (8) under the constraint of equation (3)

$$
\Phi(w)=\frac{1}{2}\|w\|^{2}
$$

Introduce Lagrange multiplier $\alpha_{i}, i=1,2, \ldots, l$ and define

$$
w(\alpha)=\sum_{i=1}^{l} \alpha_{i} y_{i} x_{i}
$$

Use Wolfe's dual theorem to transform the above problem into its dual problem:

$$
\begin{aligned}
& \operatorname{Max} W(\alpha)=\sum_{i} \alpha_{i}-\frac{1}{2} w(\alpha) \cdot w(\alpha) \\
& \text { subject to } \alpha_{i} \geq 0, \sum_{i} a_{i} y_{i}=0
\end{aligned}
$$

For the linearly inseparable training set, the relaxation variable $\xi_{i}$ can be introduced and the equation (2-8) can be rewritten as the following solution problem.

$$
\begin{aligned}
& \operatorname{Min}\left(\frac{1}{2}\|w\|^{2}+C \sum_{i} \xi_{i}\right) \\
& \text { Subject to } y_{i}\left(w \cdot x_{i}+b\right) \geq 1-\xi_{i}, \xi_{i} \geq 0
\end{aligned}
$$

Similar dual problems can be obtained:

$$
\begin{aligned}
& \operatorname{Max} W(\alpha)=\sum_{i} \alpha_{i}-\frac{1}{2} w(\alpha) \cdot w(\alpha) \\
& \text { subject to } 0 \leq \alpha_{i} \leq C, \sum_{i} a_{i} y_{i}=0
\end{aligned}
$$

Solving in the form of (10) and (12) is a typical constrained quadratic optimization problem, and there have been many mature solving algorithms.

Given a set of independent and identically distributed labeled training sample points

$$
\left(x_{1}, y_{1}\right), \ldots,\left(x_{n}, y_{n}\right), x_{i} \in R^{m}, y_{i} \in\{-1,+1\}
$$

And another set of unlabeled sample points from the same distribution

$$
x_{1}^{*}, x_{2}^{*}, x_{3}^{*}, \ldots, x_{k}^{*}
$$

Under the general linear inseparable conditions, the training process of $T$. Joachims' direct push vector machine can be described as the following optimization problem:

$$
\begin{aligned}
& \text { Minimize over }\left(y_{1}{ }^{*}, \ldots, y_{k}{ }^{*}, w, b, \xi_{1}, \ldots, \xi_{n}, \xi_{1}^{*}, \ldots, \xi_{k}{ }^{*}\right) \\
& \begin{aligned}
& \frac{1}{2}\|w\|^{2}+C \sum_{i=1}^{n} \xi_{i}+C^{*} \sum_{j=1}^{k} \xi_{j}{ }^{*} \\
& \text { subject to }: \forall_{i=1}^{n}: y_{i}\left[w \cdot x_{i}+b\right] \geq 1-\xi_{i} \\
& \forall_{j=1}^{k}: y_{j}\left[w \cdot x_{j}{ }^{*}+b\right] \geq 1-\xi_{j}^{*} \\
& \forall_{i=1}^{n}: \xi_{i} \geq 0 \\
& \forall_{j=1}^{k}: \xi_{j}^{*} \geq 0
\end{aligned}
\end{aligned}
$$

The parameters $C$ and $C^{*}$ are the parameters 
specified and adjusted by the household. The function of parameter $\mathrm{C}$ in formula (11) is similar. The parameter $C^{*}$ is called the influence factor of the unlabeled sample in the training process, and $C^{*} \xi_{j}^{*}$ is called the influence term of the unlabeled sample $\mathrm{j}$ in the objective function.

The training process of TSVM is also the process of solving the above optimization problem.

\subsubsection{Classification model of hidden dangers based} on support vector machine. Divide the safety hazard sample data into two categories: training data and test data. Among them, the training data is used to train the SVM classification model, and the test data is used to test the classification effect of the trained SVM model. Input vectorized safety hazard test data and classification model set, the classifier can determine the category of the safety hazard event. Through the model, it can be determined which type of security risk common event the input case belongs to.

The classification model is mainly composed of three parts: text training, text classification and classification evaluation model.

A. Text representation. Through the learning of the training safety hazard data set, the model can obtain a set of mutual information values of the following data words. The mutual information value of the words expresses the belonging relationship between the categories, so a very important work in text training is to calculate the word Mutual information value. In addition, only after expressing the text as a vector space model, the classifier can classify, through learning the training text set, a set of mutual information values of words is obtained, and the vectorization of the system is based on the set of mutual information values of words .

B. Text classification. Based on the SVM classification algorithm, input vectorized safety hazard text information and classification model set, the classifier can determine the category of the safety hazard event. This situation is common: an event can belong to multiple categories in the category system. However, considering the rationality of the performance evaluation of the classification results, the classification system is designed as a single classifier, that is, the text of the safety hazard event is eventually assigned to only one category.

C. Evaluation model. The classification evaluation model part evaluates the performance of the classifier, and at the same time gives feedback information to learn, so as to continuously modify the classification characteristics. Finally, the classification result is returned to the user, and the user confirms or corrects the classification result, so as to realize the self-learning of the classifier features and improve the classifier's ability to classify hidden safety events.

\section{Establishing a library of measures to deal with hidden dangers in power systems}

\subsection{Distribution characteristics of common safety hazards}

Text segmentation, text clustering and other methods are used to identify and extract the characteristic information of common security risks. On this basis, and based on statistical methods to determine the multi-dimensional distribution characteristics of each type of safety hazard event and its associated factor set, establish a common set of safety hazard event feature set, in order to achieve automatic classification of safety hazards, and provide information basis for the subsequent efficient work of safety hazard investigation .

After collecting data on 140 potential hazards in the power system, and processing the classification of hidden hazards, it can be analysed that 140 hidden hazards are evenly distributed in 14 categories of projects. As shown in Figure 2, there are no prominent hidden hazard categories among the 14 categories. All hidden hazards need to be strictly guarded against.

\section{Hidden hazard classification ratio chart}
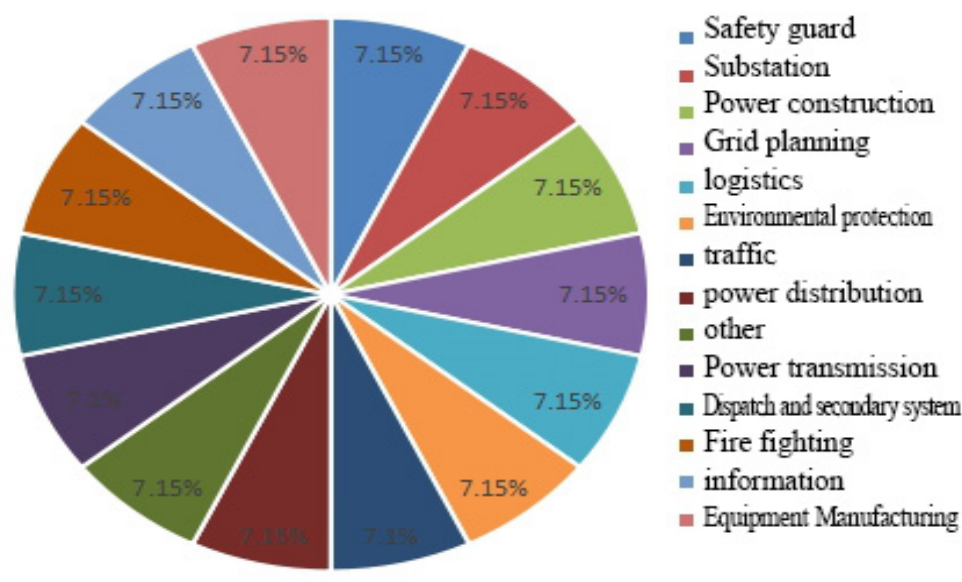

Figure 2. Proportion of all hidden hazard professional classifications 


\subsection{Treatment measures library}

The general structure of the expert system is composed of six parts: knowledge base, inference engine, comprehensive database, human-machine interface, interpreter and knowledge acquisition program, as shown in Figure 3.

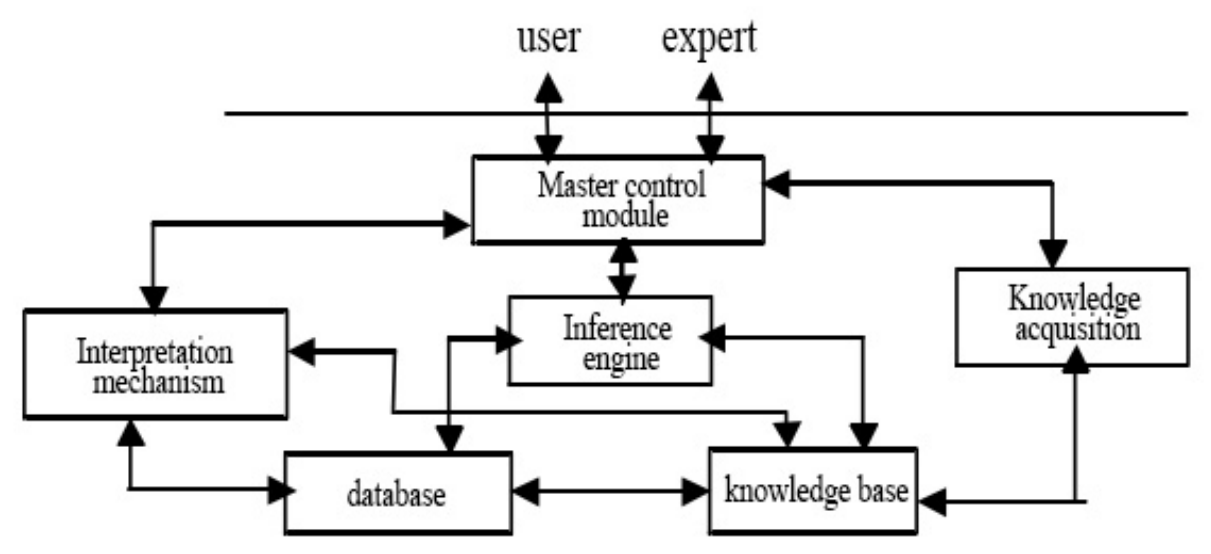

Figure 3. Treatment measures library structure

Knowledge base refers to a collection of processing measures expressed in a certain form stored in the expert system; the inference engine is a set of programs that controls and coordinates the work of the entire system; the interpretation system is mainly used to explain the inference results of the system itself and answer the user's problems. The basic structure of the expert system is mainly composed of a production structure, which defines the specific rules as: IF (condition) THEN (conclusion).

The most distinctive feature of an expert system is that it possesses the knowledge of experts in certain fields. The knowledge base is one of the most basic components of the expert system. The choice of knowledge representation in the knowledge base has become a very important issue. There are two main requirements for knowledge representation[15]. On the one hand, it should be as close as possible to the form used by domain experts in their work and thinking process, which is conducive to improving the accuracy of experience representation and the ease of knowledge coding; on the other hand, Must also consider the storage space and running time savings and how to improve the efficiency of the expert system.

According to the above frameworks, text segmentation, text clustering and other methods are used to identify and extract the characteristic information of common safety hazard events, based on statistical methods to determine the multi-dimensional distribution characteristics of each type of safety hazard events and their associated factor sets, to establish a common safety hazard event feature set, to realize automatic classification of safety hazards, and then through algorithms such as text word segmentation to extract its characteristic information, and applying support vector machine classification model to determine which type of common event this case belongs to. Finally, all the measures for this event in the safety supervision and management system are statistically obtained, and applied. Semantic analysis, word segmentation clustering and other methods determine the representative treatment measures of the event; express them as the production knowledge items and add them to the treatment measure database, finally completing the construction of the safety hazard treatment measure database.

\section{Conclusion}

In this paper, based on the domestic and foreign big data technology, security risk data semantic analysis and other related research results, combined with the characteristics of the power company intranet, the code of the security risk data is analyzed.. This paper proposes an automatic classification model of safety hazards based on SVM. Building a library of standard safety hazard treatment measures provides scientific decision-making measures for safety hazard management. It can effectively solve the problem of insufficient security data entry and inconsistent semantic expression, and realize the in-depth mining and utilization of security risk data.

\section{References}

1. Vassiliadis P, Simitsis A, Skiadopoulos S. (2002) Conceptual Modeling for ETL Processes. In: Proceedings of the 5th ACM International Workshop on Data Warehousing and OLAP. New York: ACM. pp. 14-21.

2. Simitsis A. (2005) Mapping Conceptual to Logical Models for ETL Processes. In: Proceedings of the 8th ACM International Workshop on Data Warehousing and OLAP. New York: ACM. pp. 67-76.

3. Calvanese D, Giacomo G D, Lenzerini $M$, et al. (1999) A Principled Approach to Data Integration and Reconciliation in Data Warehousing. In: Proceedings of the International Workshop on Design and Management of Data Warehouses. 
Picataway, NJ: DMDW. pp. 16.

4. Sheth A P. (1998) Changing Focus on Interoperability in Information Systems: From System, Syntax, Structure to Semantics. In: Interoperating Geographic Information Systems[C]. Norwell, MA: Kluwer Academic Publisher. pp. 5-30.

5. Hoang A D T. (2008) An Integrated Use of CWM and Ontological Modeling Approaches towards ETL Processes. In: Proceedings of the 2008 IEEE International Conference on e-Business Engineering. Picataway, NJ: IEEE. pp. 715-720.

6. Monge A E. (2000) Matching Algorithms within a Duplicate Detection System. J. IEEE Data Engineering Bulletin, 23(4):14-20.

7. Marcus A E, Maletic J I, Lin K L. (2001) Ordinal Association Rules for Error Identification in Data Sets. In: Proceedings of the 10th International Conference on Information and Knowledge Management. New York: ACM. pp. 589-591.

8. Inmon W H, Conklin E. (1994) Loading Data into the Warehouse. J. Tech Topic, ,11(1):20-25.

9. Chen Guilin, Wang Yongcheng. (2000) An improved fast word segmentation algorithm. J. Computer Research and Development, 37(4):418-424.

10. Su Fangzhong, Lin Shiping. (2004) Research and Implementation of a Chinese Word Segmentation Algorithm in Web Text Mining. J. Journal of Fuzhou University, 32(s1):67-71.

11. Sun Boya. (2011) Application Research of Chinese Word Segmentation Algorithm in GIS [D]. Central South University.

12. Gil-García R, Pons-Porrata A. (2008) Hierarchical Star Clustering Algorithm for Dynamic Document Collections. J.

13. Chen C L, Tseng F S C, Liang T. (2010) Mining fuzzy frequent itemsets for hierarchical document clustering. J. Information Processing \& Management, 46(2):193-211.

14. Li Y, Chung S M, Holt J D. (2008) Text document clustering based on frequent word meaning sequences. J. Data \& Knowledge Engineering, 64(1):381-404.

15. Bonabeau E, Dorigo M, Theraulaz G. (1999) Swarm intelligence: From natural to artificial systems. New York: Oxford University Press. pp. 40-58. 